\title{
Reliable multipoint temperature profiling in hydroprocessing units
}

\author{
$\underline{S t e f a n o ~ M e l l a ~}^{1}$, Amar Löffler ${ }^{2}$, Marc Schalles $^{3}$ \\ ${ }^{1}$ Endress+Hauser Temperature and System Products, Pessano con Bornago, Italy \\ ${ }^{2} 3$ Endress+Hauser Temperature and System Products, Nesselwang, Germany \\ stefano.mella@endress.com
}

\begin{abstract}
Summary:
Multipoint thermocouple probes are frequently used in oil and gas industry and chemical applications to detect temperature profiles. Because of the critical process conditions inside the vessels, the sheaths of such probes might be affected by crack phenomena. Due to that the process fluid might penetrate through the sheath, causing electrical insulation drops, measurement drift and in the worst case overall short circuits. A new thermometer design aims to be a more robust solution thanks to its double sealing layers, double insulation layers and completely independent sensors.
\end{abstract}

Keywords: industrial thermometer, profile temperature, advanced measurement, multi-thermocouple cable

\section{Introduction}

In the oil and gas refining industry, catalytic hydroprocessing units such as hydrotreaters (HDT) or hydrocracker units (HCU) rely on highperformance catalyst technologies to maximize product conversion while efficient reaction control seeks to keep the environmental footprint and cost down. Precise and reliable temperature mapping of densely packed reactor catalyst beds therefore makes an essential contribution to stable and profitable unit operations.

Multipoint temperature instruments with thermocouple sensors are widely used in the industry as they monitor optimum heat distribution, preventing hotspots and premature catalyst deactivation under high-temperature, highpressure and corrosive conditions. However, most conventional multipoint thermocouple probe designs have two major weaknesses:

- Reliability: A phenomenon known as hydrogen sulfide (H2S) contamination affects conventional magnesium oxide ( $\mathrm{MgO})$ cables under extreme process conditions. H2S contamination can alter measurement accuracy or even lead to a loss of control over the reaction with potentially disastrous consequences.

- Size: They are comparatively invasive, taking up valuable space in catalyst beds, leading to undesired pressure drops and channeling effects.

Standard thermocouple sensors are embedded in insulating magnesium oxide ( $\mathrm{MgO}$ ) powder and surrounded by a stainless steel sheath, providing some level of protection. However, under extreme conditions, even microscopic cracks form in the outer sheath allow hydrogen sulfide to permeate into the MgO powder, causing detrimental contamination of the cable internals that may go unnoticed by operators [1].



wires

Fig. 1. Cross section of typical mineral insulated Thermocouple

Ageing mechanisms: Hydrogen stressinduced cracking (HSC)

It is standard practice to bend (route) sensor cable probes inside a reactor according to the required layout. This flexible installation ensures that measurement points are adequately distributed, for example across a catalyst bed, to provide the desired temperature profile. However, the bending of metal induces expansion and compression stress, causing weak spots along sharp bends in particular.

In hydrogen-rich atmospheres, HSC may occur at such spots, which in time can grow enough 
to break through the metal sheath entirely [2]. This loss of integrity leads to larger molecules of the process fluid (e.g. H2S) reaching inside the insulating $\mathrm{MgO}$ powder, contaminating it.

\section{Ageing mechanisms: H2S contamination}

Magnesium oxide powder reacts with certain chemicals, including sulfur and nickel. The now contaminated $\mathrm{MgO}$ powder enables the formation of electrically conductive Ni3S2 by combining nickel from the thermocouple conductor leads, the metal sheath and sulfur from the process. This can be seen in Fig. 2 to 4 . Here, the contaminated area 5 grows, the electrical leads form a short circuit, restricting the sensor accuracy or migrating the location of the thermocouple hot junction. The probe risks becoming blind to process temperature changes.

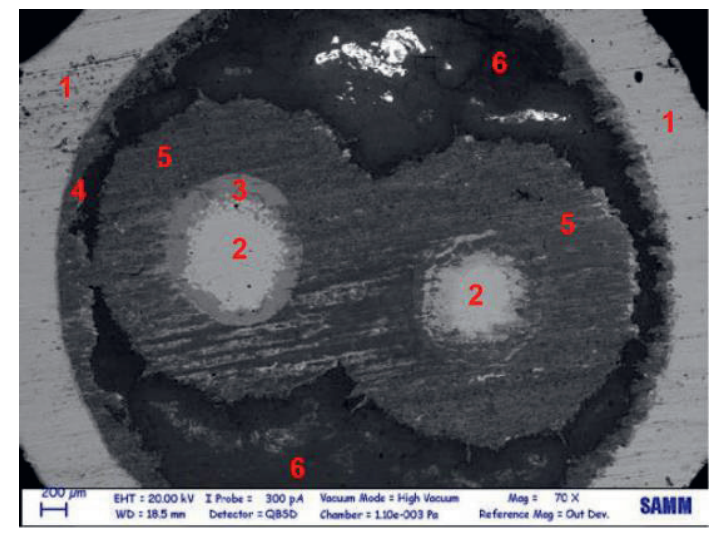

1 - Outer sheath (standard thermocouple)

2 - Thermocouple legs $(+/-)$ kernels

3 - Thermocouple legs (+/-) polluted crowns

4 - Nickel migration from the outer sheath

5 - MgO powder, polluted by $\mathrm{Ni}$ and $\mathrm{S}$

6 - Magnesium or aluminium powder

Fig. 2. Electron microscopy scan of a cross section of a standard mineral insulated sheathed thermocouple of Type $N$

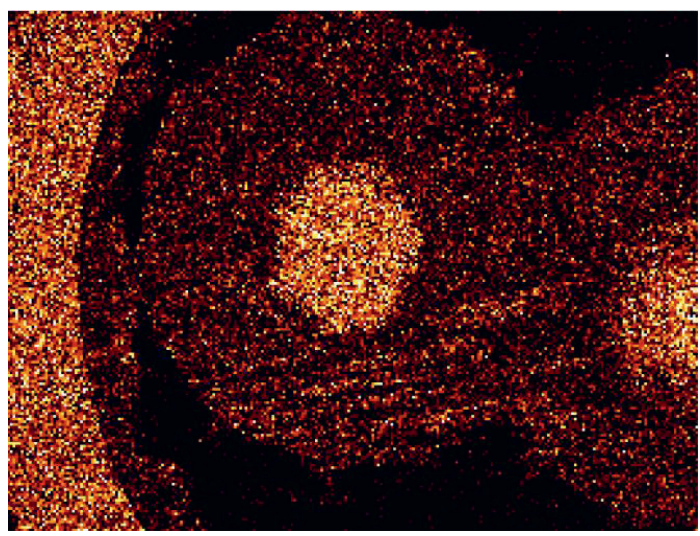

Fig. 3. Distribution of Nickel

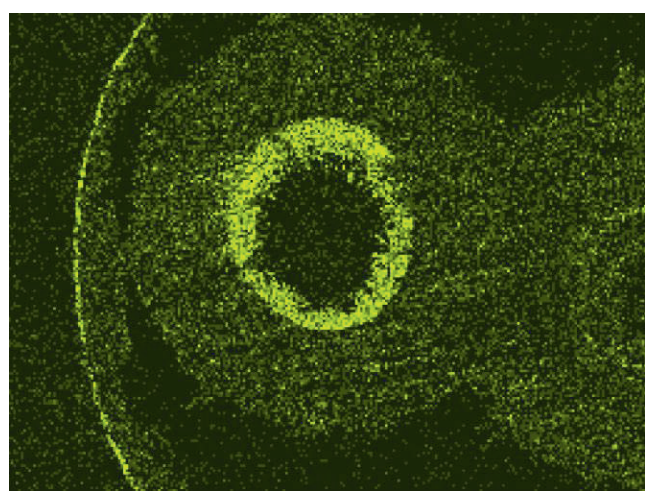

Fig. 4. Distribution of Sulfur

\section{Description of the new thermocouple design}

To provide a higher process safety, a new, robust multipoint thermocouple probe design was developed. It combines a thermowell and multiple thermocouple sensors in a single spacesaving probe (Fig. 5). Here the thermowell/ outer sheath provides a first process barrier. In case of its leakage the internal $\mathrm{MgO}$ powder could be polluted. But, this does not distort the thermocouple measurement since each of the thermocouples is surrounded by a second sheath. This is a second layer of protection to thermocouple measuring systems.

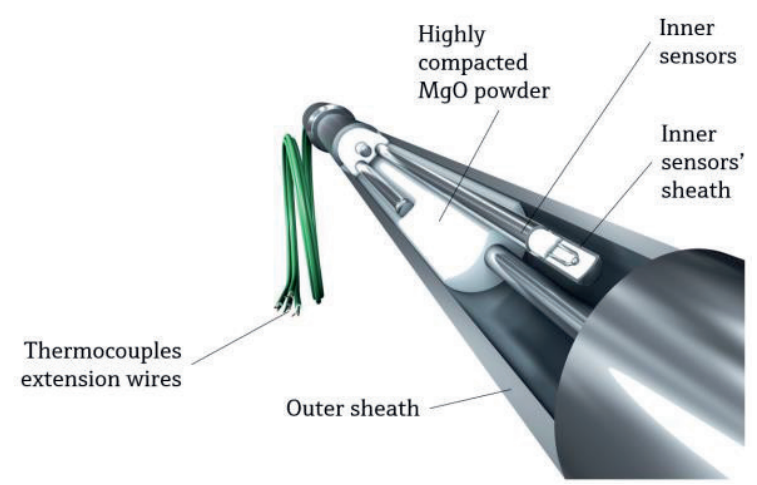

Fig. 5. Sketch of the new designed ProfileSens

Multiple thermocouple sensors can be grouped within a single probe, each delivering ultra-high measuring performance even under the most challenging operation conditions. The probe layout (routing), length and the number of sensors is individually adapted to process specifications. The design is proven in use to significantly lower the risk of premature sensor drift, corrosion or short circuits.

\section{References}

[1] E. S. Webster, Low-Temperature Drift in MIMS Base-Metal Thermocouples, International Journal of Thermophysics 35, 574-595 (2014); doi: $10.1007 / \mathrm{s} 10765-014-1581-9$

[2] M. S. Rana et. Al., Degradation of thermocouple in a temperature programmed sulphidation reactor, Eng. Fail. Anal. 55, 79-86 (2015); doi: 10.1016/j.engfailanal.2015.05.012 